\title{
SLIT: Daten zur Kosten-Nutzen-Analyse
}

\section{Die Implementierung therapeutischer Innovationen erfordert heute neben dem Nachweis der klinischen Wirksamkeit auch einen Nachweis des Gesamtnutzens der Therapieform im Rahmen des Gesundheitswesens. Dazu gehören auch Daten aus Studien unter den Bedingungen des tatsächlichen Praxisalltags, - nun untersucht für die sublinguale spezifische Immuntherapie.}

$\mathrm{H}$ ealth Technology Assessment (HTA) ist ein Instrument zur Erfassung von Effektivität und Ökonomie einer medizinischen Intervention, das zur gesundheitsökonomischen Gesamtbewertung eines Behandlungsverfahrens genutzt wird. Dabei werden Ergebnisse zu Morbidität, Mortalität, Lebensqualität, Sicherheit und Kosten im Zusammenhang mit der Intervention aus verschiedenen Datenquellen systematisch zusammengetragen und übergreifend bewertet. Ein HTA der SLIT steht noch aus. Deshalb wurde die SIMAP-Studie (Sublingual Immunotherapy in Allergic Patients) ins Leben gerufen, bei der allergologisch tätige Zentren mit einer speziellen Software zur Erfassung von pharmakoökonomischen Daten ausgestattet wurden. Zunächst wurden in einem prospektiven longitudinalen Design Informationen zu Diagnose und Behandlung von Patienten mit Atemwegsallergien gesammelt. Aus dem kompletten Datensatz eines Jahres erfolgte eine nähere Analyse von 102 Patienten mit einer Gräserpollenallergie. Eine Gruppe war lediglich symptomatisch, die andere mit einer GräserpollenSLIT (Staloral 300) und zusätzlich nach Bedarf symptomatisch behandelt worden. Es zeigte sich eine Reduktion der Bedarfsmedikation bei immuntherapierten Patienten im Vergleich zu den
Kontrollen. Die jährlichen Gesamtkosten lagen bei SLIT mit durchschnittlich 311 Euro im Vergleich zur alleinigen symptomatischen Therapie mit 180 Euro höher. Von den Symptomatika konnten aber durch die SLIT 22\% der Kosten bei Patienten mit einer Rhinitis und 34\% der Kosten bei Patienten mit Rhinitis und Asthma eingespart werden.

Fazit: Bereits im ersten Behandlungsjahr ergeben sich unter den Bedingungen des Praxisalltags durch eine SLIT Einsparungen von Symptomatika. Die Kosten für die Immuntherapie sind in diesem Zeitraum allerdings höher als unter alleiniger symptomatischer Therapie. Zur Bewertung des Gesamtnutzens müssen nun Daten z. B. zur Lebensqualität und zum weiteren Rückgang der Verordnungen von Symptomatika in den Folgejahren ermittelt werden.

bk

Berto $P$ et al. Comparison of costs of sublingual immunotherapy and drug treatment in grass-pollen induced allergy: results from the SIMAP database study. Curr Med Res Opin 2008; 24: 261-6

\section{Orale Kuhmilch-Desensibilisierung}

\section{Die Wirksamkeit einer oralen Desensibilisierung mit Milch ist nur in Untersuchungen mit kleiner Fallzahl nachgewiesen. Mittlerweile liegen dazu Langzeitdaten mit einer Beobachtungszeit von knapp fünf Jahren vor.}

V or rund fünf Jahren führten Paolo Meglio et al. vom San-Pietro-Krankenhaus in Rom eine Studie durch, an der 21 Kinder mit einer Kuhmilch-Allergie teilnahmen. Die Kinder wurden zu Hause nach einem genauen Protokoll oral mit verdünnter Milch desensibilisiert. Diese Therapie war bei $85 \%$ der Kinder ganz oder teilweise erfolgreich [Meglio P et al., 2004]. Nach vier Jahren und acht Monaten wurden 20 der ursprünglich 21 Studienteilnehmer nochmals befragt und untersucht. 13 von ihnen vertrugen Kuhmilch problemlos, ein Kind tolerierte die Milch mit Einschränkungen. Bei keinem einzigen Kind war es zu einer Notaufnahme im Rahmen einer allergischen Reaktion gekommen.
Die Reaktionen im Pricktest gegenüber Casein oder Alpha-Laktalbumin waren signifikant rückläufig; die Sensitivität hatte sich gegenüber der Zeit nach der sechsmonatigen Desensibilisierungspha-

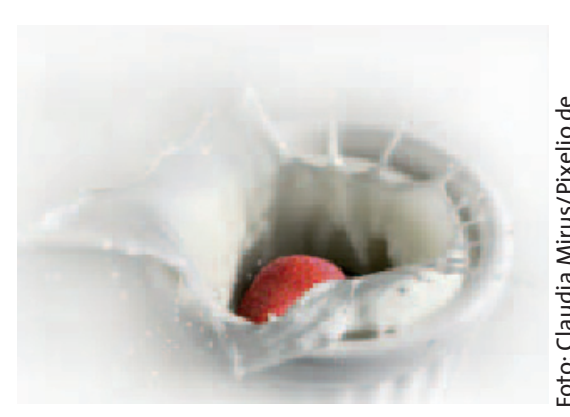

Häufig lässt sich Milchtoleranz erreichen. se nicht signifikant verändert. Unmittelbar vor und nach der oralen Desensibilisierung unterschieden sich die Spiegel des spezifischen IgE für Milchproteine nicht signifikant. Nach fünf Jahren lagen die Medianwerte aber signifikant niedriger als zu Therapiebeginn (Casein: 0,23 vs. $1,0 \mathrm{kU} / \mathrm{l}, \mathrm{p}=0,043$; Alpha-Lactalbu$\min : 0,6$ vs. $4,2 \mathrm{kU} / \mathrm{l}, \mathrm{p}=0,023$ )

Fazit: Die orale Desensibilisierung mit Kuhmilch war in der vorliegenden Untersuchung bei einem Großteil der Kinder erfolgreich. Nach knapp fünf Jahren war der Therapieeffekt meist erhalten geblieben. Die Hauttestreaktionen veränderten sich im Zeitverlauf nicht, das spezifische IgE nahm signifikant ab. Eine kontrollierte Studie mit größerer Fallzahl wäre wünschenswert.

Meglio P et al. Oral desensitization in children with immunoglobulin E-mediated cow's milk allergy - follow-up at $4 \mathrm{yr}$ and 8 months. Pediatr Allergy Immunol 2008 Jan 22 [Epub ahead of print]. 